\title{
Factors Influencing Time Resolution of Scintillators and Ways to Improve Them
}

\author{
P. Lecoq, Member, IEEE, E. Auffray, Member, IEEE, S. Brunner, H. Hillemanns, Member, IEEE, \\ P. Jarron, Member, IEEE, A. Knapitsch, T. Meyer, and F. Powolny
}

\begin{abstract}
The renewal of interest in Time of Flight Positron Emission Tomography (TOF-PET), as well as the necessity to precisely tag events in high energy physics (HEP) experiments at future colliders are pushing for an optimization of all factors affecting the time resolution of the whole acquisition chain comprising the crystal, the photo detector, and the electronics. The time resolution of a scintillator-based detection system is determined by the rate of photo electrons at the detection threshold, which depends on the time distribution of photons being converted in the photo detector. The possibility to achieve time resolution of about 100 ps Full Width at Half Maximum (FWHM) requires an optimization of the light production in the scintillator, the light transport and its transfer from the scintillator to the photo detector. In order to maximize the light yield, and in particular the density of photons in the first nanosecond, while minimizing the rise time and decay time, particular attention must be paid to the energy transfer mechanisms to the activator as well as to the energy transition type at the activator ion. Alternatively other light emission mechanisms can be considered. We show that particularly Cerenkov emission can be used for this purpose. Special emphasis was put on the light transport within the crystal and at its interface with the photo detector. Since light is produced isotropically in the scintillator the detector geometry must be optimized to decrease the optical path-length to the photo detector. Moreover light bouncing within the scintillator, affecting about $70 \%$ of the photons generated in currently used crystals, must be reduced as much as possible. We also investigate photonics crystals that are specifically designed to favor specific light propagation modes at the limit of total reflection inside and outside of the crystal and how they might increase the light transfer efficiency to the photo detector and hence improve time resolution. Examples for the production and deposition of photonics crystals as layers on Lutetium Yttrium Ortho-Silicate (LYSO) and Lutetium Yttrium Aluminum Perovskite (LuYAP) crystals are shown here, as well as first results on an improved light extraction resulting from this method.
\end{abstract}

Index Terms-Cerenkov, PET, photonic crystal, photostatistics, time-of-flight, timing resolution.

\section{INTRODUCTION}

D URING the last two decades a number of new, bright, fast, and radiation hard scintillators has emerged and been used in large scale particle physics experiments, like Compact

Manuscript received November 09, 2009; revised March 03, 2010; accepted April 21, 2010. Date of publication July 01, 2010; date of current version October 15,2010 .

The authors are with CERN, 1211 Geneva 23, Switzerland (e-mail: paul. lecoq@cern.ch).

Color versions of one or more of the figures in this paper are available online at http://ieeexplore.ieee.org.

Digital Object Identifier 10.1109/TNS.2010.2049860
Muon Solenoid (CMS) and ALICE at CERN, as well as in commercial or prototype positron emission tomographs (PET) scanners. Crystal calorimeters in particle physics typically employ, e.g., pure, Na- or Tl-doped cesium iodide (CsI) crystals and lead tungstate $\left(\mathrm{PbWO}_{4}\right)$ crystals, whereas commercial or prototype tomographs are usually made of lutetium ortho-silicate (LSO), lutetium aluminum ortho-silicate (LYSO), or lanthanum bromide $\left(\mathrm{LaBr}_{3}\right)$ crystals.

So far the main requirements in this domain have been on high material density to increase conversion efficiency, but also high energy and position resolution, with a demand for fast light decay to allow high counting rates, while keeping the dead time low.

The necessity to precisely tag events in high energy physics (HEP) experiments at future colliders, where high luminosity is achieved through densely spaced bunch trains, as well as new requirements in PET for improved signal-to-noise ratio $(\mathrm{S} / \mathrm{N})$ images, lower exposure rates for patients and significantly faster image reconstruction, were the driving elements in our search for higher time resolution than currently achievable. Therefore our efforts focused on the optimization of every parameter that could possibly influence the timing precision in a PET detector and its data acquisition chain.

The majority of commercial PET cameras do not use time-offlight (TOF) information and are therefore limited to timing resolutions at the level of $\sim 2 \mathrm{~ns}$. On the other hand, following the development of fast and high-light-yield crystals such as LSO:Ce [1], PET systems with TOF capability have received new attention. The only commercial systems developed so far with this feature are the Philips "TruFlight" with 650 ps FWHM and new devices from Siemens with 550 ps and from General Electric with 590 ps FWHM temporal resolution. This corresponds to a spatial resolution of approximately $9 \mathrm{~cm}$ along the line of response (LOR), only sufficient to reduce the loss in image quality aggravated by the size of obese patients. Despite being a significant improvement over standard PET cameras, this precision does not yet allow a direct 3-D reconstruction of small and medium sized objects or organs. Only a target value of 100 ps to 200 ps FWHM in timing resolution would lead to a spatial precision of $1.5 \mathrm{~cm}$ to $3 \mathrm{~cm}$ along the LOR, allowing the localization of the organ under examination (e.g., prostate, pancreas or lymph nodes) and an efficient rejection of the background generated by other organs. In fact such a precision has the additional potential to allow a direct $3 \mathrm{D}$ reconstruction and to eliminate the time consuming iterative or back-projection algorithms. Also hadron therapy would greatly benefit from a fast on-line monitoring of the dose delivered during proton or carbon 
therapy treatment, requiring very high sensitivity, high resolution and fast reconstruction imaging of $\beta^{+}$emitting isotopes produced by beam or target spallation processes during the irradiation [2].

Such a challenging objective can only be achieved through an in-depth understanding and optimization of the light production and light transport mechanisms in the crystal, which we will describe in the next paragraphs.

\section{Light PRODUCTION}

\section{A. Theoretical Considerations}

The time resolution of a scintillator-based detector is directly driven by the density of photo electrons generated in the photo detector at the detection threshold. At the scintillator level it is related to the intrinsic light yield, the pulse shape (rise and decay time) and the light transport from the production point to the photo detector. The well understood relaxation scheme of electronic excitations in a scintillator shows that the critical part for timing is the localization and trapping of electrons and holes by the activator ions at the end of the thermalization process. The presence of traps can induce delayed charge transfer processes and hence increase the rise time. On the other hand, the decay time is driven by the time of optical transition in the activator. Allowed electric dipole transitions at high optical energy (short wavelength) must be favored as the probability $\Gamma$ of radiative transitions in a medium with index of refraction $n$, inversely proportional to the decay time $\tau_{\mathrm{sc}}$, is proportional to the oscillator strength $\mu$ of the transition between the initial state $i$ and the final state $f$ :

$$
\sum_{f}|\langle f|\mu| i\rangle|^{2}
$$

and inversely proportional to the 3rd power of the emission wavelength $\lambda_{\text {em }}[3]$ :

$$
\Gamma=\frac{1}{\tau_{\mathrm{sc}}} \propto \frac{n}{\lambda_{\mathrm{em}}^{3}}\left(\frac{n^{2}+2}{3}\right)^{2} \sum_{f}|\langle f|\mu| i\rangle|^{2}
$$

Non-radiative trapping, photo ionization, thermal or concentration quenching can shorten this decay time but at the expense of a reduction in light yield. Whereas this might be acceptable for high energy experiments, it is not desirable for energies used in medical physics where the timing properties of all commonly used scintillators are still strongly limited by photo statistics. Indeed the time fluctuation $t_{j}$ of the $n$th photo electron for a scintillator producing a total light output of $R$ photo electrons with a decay time $\tau$ is given by [4]:

$$
t_{j}=\tau \ln \left(\frac{1}{2}\left(n-R+\sqrt{(n-R)^{2}+4 R}\right)\right)
$$

The actual timing resolution results from the combined probability that the $(n-1)$ th photo electron has been detected at time

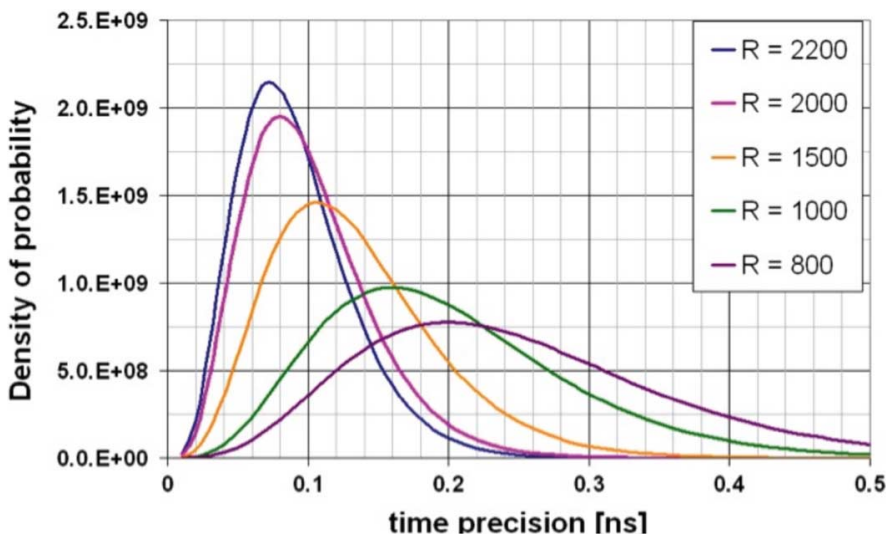

Fig. 1. Density of probability for the detection time of the 5 th photo electron of a scintillator with a 40 ns decay time for several values of light output $R$.

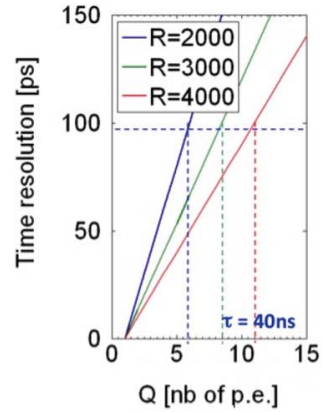

a)

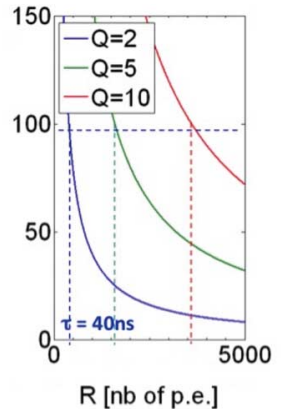

b)

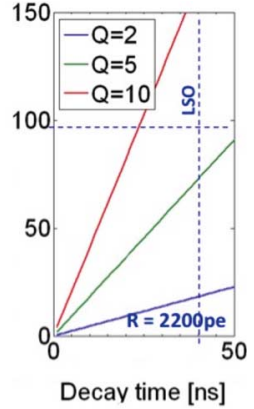

c)
Fig. 2. Timing resolution dependence on a) the detection threshold Q, b) the light output $\mathrm{R}$ and c) the decay time $\tau$ for three different light outputs and detection threshold values.

$\mathrm{t}$ and the $\mathrm{nth}$ one is detected between $\mathrm{t}$ and $\mathrm{t}+\mathrm{dt}$, integrated for all photo electrons between the first and the nth one. The result is illustrated on Fig. 1 for a scintillator with 40 ns decay time, a detection threshold of five photo electrons and a light yield $R$ varying between 800 and 2200 photo electrons, illustrating the importance of a high light yield for an improved timing resolution. The value of 2200 photo electrons for the light output derives from our measurement of 1000 pixels of LYSO $(2 \times 2 \times 20$ $\mathrm{mm}^{3}$ ) produced for the ClearPEM-Sonic project and measured with a Photonis 2020Q photomultiplier [5]

Fig. 2 shows the limits on the detection threshold expressed in terms of photo electrons for three different values of light output, as well as the light output in units of photo electrons and the decay time in ns for three different values of the number of photo electrons at the detection threshold, to reach a 100 ps timing resolution.

It should be noted that these curves have been obtained from the Poisson distribution of photo electrons produced in a scintillator with a mono-exponential decay time and no rise time. Table I shows how a short rise time (1 ns or even less) would dramatically affect the arrival time of the first 10 photo electrons, as already pointed out in [6].

These numbers show that there is a clear advantage for choosing the lowest possible detection threshold. The ideal condition would be a fully digital photon counting detector with a precise time stamp for each optical photon detected. 
TABLE I

ARrival TIME of the First 10 PHOto EleCtrons FOR LYSO (2200 Detected Photo Electrons, 40 ns Decay Time) FOR FOUR RISE TIME CONDITIONS

\begin{tabular}{ccccc}
\hline \multirow{2}{*}{$\begin{array}{c}\text { Photo } \\
\text { EleCtrons }\end{array}$} & \multicolumn{4}{c}{ Rise Time [ns] } \\
& 0 & 0.2 & 0.5 & 1.0 \\
\hline \hline 1 & $17 \mathrm{ps}$ & $92 \mathrm{ps}$ & $134 \mathrm{ps}$ & $198 \mathrm{ps}$ \\
2 & $35 \mathrm{ps}$ & $134 \mathrm{ps}$ & $200 \mathrm{ps}$ & $279 \mathrm{ps}$ \\
3 & $55 \mathrm{ps}$ & $169 \mathrm{ps}$ & $252 \mathrm{ps}$ & $352 \mathrm{ps}$ \\
4 & $72 \mathrm{ps}$ & $198 \mathrm{ps}$ & $293 \mathrm{ps}$ & $403 \mathrm{ps}$ \\
5 & $91 \mathrm{ps}$ & $225 \mathrm{ps}$ & $334 \mathrm{ps}$ & $452 \mathrm{ps}$ \\
6 & $109 \mathrm{ps}$ & $252 \mathrm{ps}$ & $359 \mathrm{ps}$ & $501 \mathrm{ps}$ \\
7 & $126 \mathrm{ps}$ & $277 \mathrm{ps}$ & $403 \mathrm{ps}$ & $546 \mathrm{ps}$ \\
8 & $145 \mathrm{ps}$ & $300 \mathrm{ps}$ & $435 \mathrm{ps}$ & $588 \mathrm{ps}$ \\
9 & $164 \mathrm{ps}$ & $323 \mathrm{ps}$ & $466 \mathrm{ps}$ & $627 \mathrm{ps}$ \\
10 & $183 \mathrm{ps}$ & $347 \mathrm{ps}$ & $497 \mathrm{ps}$ & $664 \mathrm{ps}$ \\
\hline
\end{tabular}

\section{B. Properties and Limits of Available Scintillators}

In order to maximize the light yield, particularly the density of photons in the first nanosecond, and minimize the light rise and decay time, special attention must be paid to the mechanisms of energy transfer to the activator as well as to the energy transition type at the activator ion. It has been clearly established in recent years that spin-allowed dipole-dipole transitions, as observed between the $5 \mathrm{~d}$ and $4 \mathrm{f}$ energy levels of $\mathrm{Ce}^{3+}$ are among the fastest and most efficient scintillation modes. As can be seen from (1) a high index of refraction and a short wavelength are the conditions for a short decay time.

However as recently pointed out by Dorenbos [7] it will probably be unlikely to reach values below the limit of $15 \mathrm{~ns}$ to $17 \mathrm{~ns}$ for $\mathrm{Ce}^{3+}$ activated scintillators. Crystals like $\mathrm{LaCl}_{3}: \mathrm{Ce}$, $\mathrm{LaBr}_{3}: \mathrm{Ce}$ and LuAP:Ce are already close to this limit. On the other hand according to (1), praseodymium $\left(\mathrm{Pr}^{3+}\right)$ with its $5 \mathrm{~d}-4 \mathrm{f}$ transition energy being $1.55 \mathrm{eV}$ higher than that of $\mathrm{Ce}^{3+}$, has the potential for a three times faster scintillation mode than cerium. Even in view of the fact that this higher energy transition shifts the emission spectrum from visible to UV, and that complex energy transfer mechanisms with the host element can give rise to a slower rise time, $\mathrm{Pr}^{3+}$ is an attractive activator for fast decay materials.

In spite of a lower light yield cross-luminescent materials produce more photons in the first nanosecond $(\sim 1100$ in case of $\left.\mathrm{BaF}_{2}\right)$ than $\mathrm{LSO}(\sim 670)$. Indeed $\mathrm{BaF}_{2}$ was the first choice for scintillator material in TOF-PET in the 1980s. Unfortunately, notwithstanding their excellent time resolution, the deep-UV emission of these cross-luminescent crystals and their low density made them inappropriate for PET applications.

Novel solutions may well come from direct gap semi-conductors or nano-crystals. By creating a donor band in the vicinity of the conduction band in a semiconductor such as $\mathrm{ZnO}$ or $\mathrm{CdS}$ a very fast sub-nanosecond band-to-band recombination process can take place. First discovered in the 1960s by Lehmann [8] this process was reintroduced by Derenzo [9] a few years ago and seems to apply also to heavier materials like $\mathrm{CuI}, \mathrm{PbI}_{2}$ and $\mathrm{HgI}_{2}$.

High quality semiconductors, e.g., GaAs, based on nano-structures have a light yield that can reach $70-80 \%$ of absorbed energy as compared to at most $15 \%$ in standard
TABLE II

Physical Parameters of Several Scintillators FOR CERENKOV PHOTON DETETION

\begin{tabular}{cccc}
\hline \hline & $\begin{array}{c}\text { REFRACTIVE } \\
\text { INDEX } \\
\mathrm{n}\end{array}$ & $\begin{array}{c}\text { FUNDAMENTAL } \\
\text { ABSORPTION } \\
{[\mathrm{nm}]}\end{array}$ & $\begin{array}{c}\text { ELECTRON } \\
\text { RANGE* } \\
{[\mu \mathrm{m}]}\end{array}$ \\
\hline \hline $\mathrm{PbWO}_{4}$ & 2.20 & 370 & 513 \\
LSO:Ce & 1.82 & 190 & 527 \\
LuAG:Ce & 1.84 & 177 & 582 \\
LuAP:Ce & 1.95 & 146 & 487 \\
\hline *
\end{tabular}

The ELECTRON RANGE is for electrons above the Cerenkov threshold

scintillators such as $\mathrm{LSO}$ or $\mathrm{LaBr}_{3}$. Moreover the emission lifetime is usually shorter than $1 \mathrm{~ns}$. These impressive performances are attributed to electron confinement, phonon confinement and optical resonances taking place in these nano-materials. Embedded in meta-structures based on polymers or heavy scintillating glasses they could complement the usual scintillation signal with a very fast light component that could be exploited for ultimate timing purposes.

\section{Detection of Cerenkov Photons for Ultimate Timing Performance}

Cerenkov detectors are widely used for particle identification and threshold detectors in high energy physics applications. Because of the fundamental property of Cerenkov radiation, which results from the transient polarization of a medium traversed by a particle travelling faster than the speed of light in that medium, it has rarely been considered for the detection of low energy $\gamma$-rays.

It is worth noticing however that the Cerenkov threshold is given by $1 /(\beta n)$, where $n$ is the refractive index of the medium and $\beta$ is the ratio of the particle velocity and the speed of light in vacuum. As the majority of heavy scintillators used in particle detectors have a high refractive index ( $\mathrm{n}=1.82$ for LSO, 1.84 for LuAG: lutetium aluminum garnet, 1.95 for LuAP) the Cerenkov threshold is easily reached for recoil electrons resulting from photo-electric interaction, as well as from the majority of Compton interactions. The range of these recoil electrons in these heavy materials is of course limited to a few hundreds of microns, but it is nonetheless sufficient to give rise to a few tens of Cerenkov photons, as illustrated in Tables II and III, where we have calculated the Cerenkov photon yield for several scintillators exposed to $511 \mathrm{KeV} \gamma$-rays by integrating (3) over the path length of the recoil electron above the Cerenkov threshold:

$$
\frac{d N}{d x}=2 \pi \alpha\left(\frac{1}{\lambda_{1}}-\frac{1}{\lambda_{2}}\right)\left(1-\frac{1}{\beta^{2} n^{2}}\right)
$$

Here $N$ is the number of photons, $\alpha$ is the fine structure constant (1/137), $\beta=v / c$ and $n$ is the refractive index of the scintillator. The integration was made between $\lambda_{1}=250 \mathrm{~nm}$ and $\lambda_{2}=600 \mathrm{~nm}$, subtracting all the doping absorption bands that could restrict this transparency window for the Cerenkov emission. The lower limit has been conservatively set to $250 \mathrm{~nm}$ 
TABLE III

Calculated Cerenkov Yield for $511 \mathrm{KeV} \gamma$-RAyS in SEVERAL SCINTILLATORS

\begin{tabular}{lccc}
\hline \hline & $\begin{array}{c}\text { CRITICAL } \\
\text { CERENKOV } \\
\left.\text { ANGLE }{ }^{\circ}\right]\end{array}$ & $\begin{array}{c}\mathrm{e}^{-} \text {ENERGY } \\
\text { THRESHOLD } \\
{[\mathrm{keV}]}\end{array}$ & $\begin{array}{c}\# \\
\text { CERENKOV } \\
\text { PHOTONS }\end{array}$ \\
\hline \hline $\mathrm{PbWO}_{4}$ & 63 & 63 & 21 \\
LSO:Ce & 57 & 101 & 15 \\
LuAG:Ce & 57 & 97 & 22 \\
LuAP:Ce & 59 & 84 & 28 \\
\hline \hline
\end{tabular}

(even when the fundamental absorption edge is at shorter wavelengths for some of these materials, giving rise therefore to an underestimate of the number of Cerenkov photons) because of the difficulty to detect photons at shorter wavelengths. We have also assumed a linear energy decrease of the electron energy along its path to calculate the useful range above the Cerenkov threshold. This simplification does not lead to an error larger than $10 \%$.

Although small, the number of Cerenkov photons emitted by the conversion of a $511 \mathrm{KeV} \gamma$-ray can help improve the timing resolution of such detectors if the photon detection threshold can be set sufficiently low. Indeed these photons are emitted quasi-instantaneously because the propagation time of the recoil electron is very small and their time spread is only determined by different transit pathways from the emission point to the photo detector.

In order to verify that Cerenkov photons induced by the conversion of $511 \mathrm{KeV} \gamma$-rays could be detected we have made a coincidence measurement between one $2 \times 2 \times 10 \mathrm{~mm}^{3}$ ceriumdoped LYSO crystal and one $2 \times 2 \times 8 \mathrm{~mm}^{3}$ non-scintillating un-doped LuAG crystal. Each crystal was wrapped in Teflon tape on five faces and installed "flat", i.e., with one of the long $\left(2 \times 10 \mathrm{~mm}^{2}\right)$ faces on a Hamamatsu $\mathrm{H} 6533$ photomultiplier with optical grease. This mount was chosen to minimize the transit time of the photons to the detector. Both crystals were excited in coincidence by a ${ }^{22} \mathrm{Na}$ source placed $8 \mathrm{~cm}$ away from each crystal. Fig. 3 shows the amplitude vs. coincidence delay scatter plot for the un-doped LuAG crystal. The clustering of events in the upper left corner, i.e., between $5 \mathrm{mV}$ and $15 \mathrm{mV}$ on the horizontal scale, is attributed to LuAG Cerenkov photons. The projection of all events on the vertical scale then yields a very sharp timing distribution compatible with a signal generated by $\geq 5$ photo electrons. The other events are attributed to random coincidences between the LYSO and dark noise generated in the LuAG photomultiplier (PMT). They correspond to single photon events. The small concentration of these events at $6.5 \mathrm{~ns}$ delay is attributed to an artifact related to the electronics setup and is under further investigation.

If one repeats the experiment and replaces the un-doped LuAG crystal with a Cerium doped, i.e., scintillating, LuAG crystal the following picture emerges (Fig. 4), where the photo peak and the Compton events are clearly seen in addition to the Cerenkov events, but with a slightly longer delay due to the
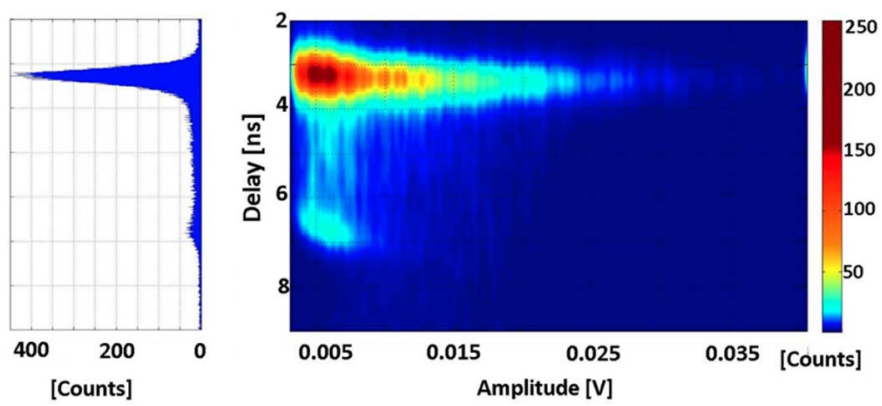

Fig. 3. Amplitude vs. coincidence-delay scatter plot for a non-scintillating un-doped LuAG crystal in an LYSO-LuAG coincidence experiment.

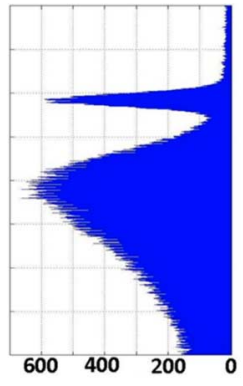

[Counts]

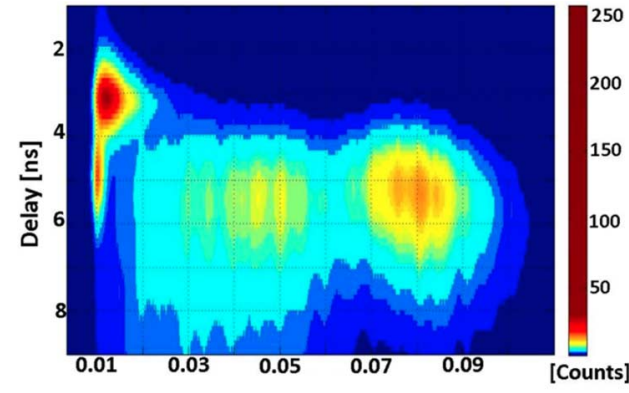

Amplitude [V]
Fig. 4. Amplitude vs. coincidence-delay scatter plot for a scintillating Ce-doped LuAG crystal in an LYSO-LuAG coincidence experiment. Note that in contrast to Fig. 3 also delayed photo electron signals from the slow decay component of the LuAG crystal are counted. The distribution is non-Gaussian because of the effect of time walk, predominantly for small signal amplitudes.

contribution of the scintillation rise and decay time. Moreover the distribution shows a fine structure corresponding to the quantification of the number of detected photo electrons.

\section{LIGHT TRANSPORT}

We have measured the LYSO coincidence timing resolution with crystals of different dimensions, against a reference LYSO crystal of $2 \times 2 \times 10 \mathrm{~mm}^{3}$ size, wrapped on five faces with Teflon tape and glued "vertically", i.e., on their small face, on two Hamamatsu H6533 PMTs. These PMTs have a rise time of $700 \mathrm{ps}$. The reference crystal timing resolution measured to be $258 \mathrm{ps}$ and the transient time spread of $160 \mathrm{ps}$ of the PMTs have been quadratically subtracted to determine the single crystal timing resolution. The results for the coincidence and individual crystal time resolutions are shown in Table IV and illustrate the strong influence of the crystal dimension and therefore of the light transit time on timing precision. This study concerns only crystals with dimensions commonly used in PET devices. Better timing resolution has been obtained with brighter crystals of shorter dimensions [10], [11].

Another key issue affecting time resolution of a scintillator is the inefficiency of light extraction from materials with a high index of refraction. This not only leads to a reduction of light collected at the photo detector, with consequences on the photo statistics described above, but also to multiple light reflections within the scintillator, which can contribute as much as $150 \mathrm{ps}$ FWHM in a $2 \times 2 \times 20 \mathrm{~mm}^{3} \mathrm{LSO}$ pixel. A possible solution to overcome the problem of total internal reflection is to improve 
TABLE IV

Time Resolutions For LYSO CRYSTALS With DimEnsions COMMONLY USED IN PET DEVICES

\begin{tabular}{cccc}
\hline \hline CRYSTAL ID & $\begin{array}{c}\text { DIMENSIONS } \\
{\left[\mathrm{mm}^{3}\right]}\end{array}$ & $\begin{array}{c}\text { COINCIDENCE } \\
\text { TIME } \\
\text { RESOLUTION } \\
\text { FWHM [ps] }\end{array}$ & $\begin{array}{c}\text { SINGLE } \\
\text { CRYSTAL } \\
\text { RESOLUTION } \\
\text { FWHM [ps] }\end{array}$ \\
\hline \hline 2023 & $3 \times 3 \times 20$ & 458 & 342 \\
2024 & $3 \times 3 \times 20$ & 446 & 325 \\
D4 & $3 \times 3 \times 15$ & 407 & 271 \\
D5 & $3 \times 3 \times 15$ & 392 & 249 \\
1123 & $2 \times 2 \times 10$ & 362 & 197 \\
\hline \hline
\end{tabular}

\section{$2.5 \mu \mathrm{m}$}

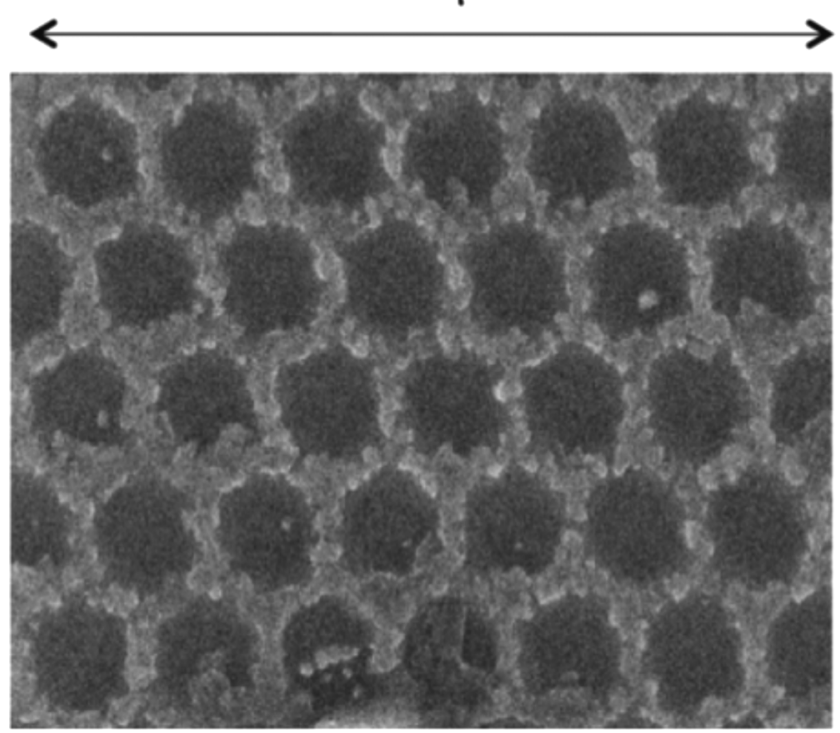

Fig. 5. Electron microscope picture of the $300 \mathrm{~nm}$ thick $\mathrm{SiN}_{4}$-based photonic crystal deposited on a LYSO Crystal.

the light extraction efficiency at the crystal/photo detector interface by means of photonic crystals (PhCs), i.e., media with a periodic modulation of the dielectric constant [12], [13].

Fig. 5 shows the structure of photonic crystals that have been deposited on $1.3 \times 2.6 \times 7 \mathrm{~mm}^{3}$ LYSO crystals using lithographic techniques on a $300 \mathrm{~nm}$ thick $\mathrm{SiN}_{4}$ layer. It is based on a triangular pattern with round holes and a pitch equal to $0.8 \lambda$.

Fig. 6 is a Monte Carlo simulation showing the angular distribution of photons arriving at the crystal/photo detector interface, as well as the distribution of extracted photons with and without the photonic crystal.

A factor of two improvement in extraction efficiency can be expected, which would allow using only the photons emitted in the direction of the photo detector with the same photo statistics as obtained by collecting all emitted photons in a crystal without "photonic" coupling. There is a clear advantage in the former configuration as the transit time spread of photons emitted in the direction of the photo- detector is much further reduced as compared to the second situation, where all the propagation modes (forward and backward) have to be used.

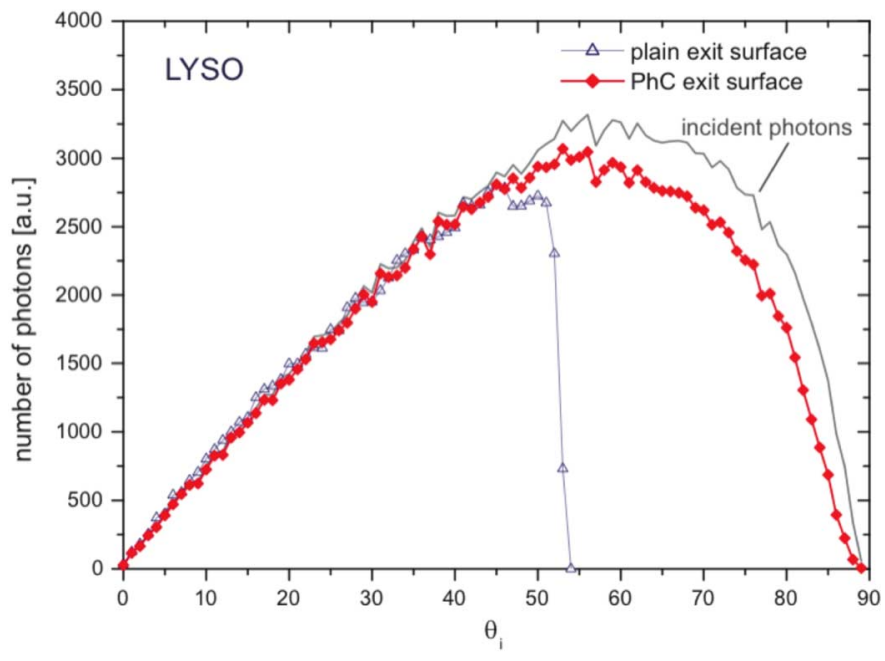

Fig. 6. Angular distribution of incoming and extracted photons in a LYSO crystal with and without a photonic crystal. From [11].

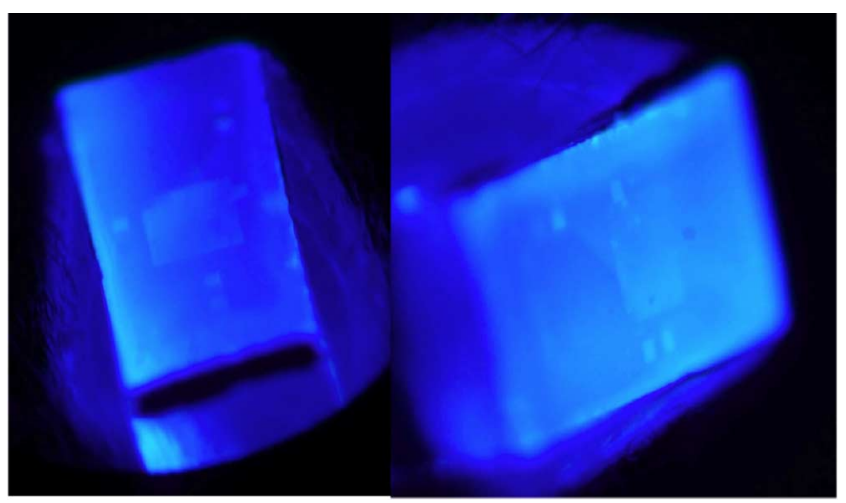

Fig. 7. Picture at two different incidence angles of the face of a LYSO crystal, on which a $300 \mathrm{~nm}$ thick photonic crystal was deposited on some parts of the surface. The crystal is excited from the side by two UV emitting LEDs.

Several photonic crystals have been designed and deposited on a central area of $600 \times 600 \mu \mathrm{m}^{2}$ on LYSO and LuYAP crystals as well as on smaller areas of $100 \times 100 \mu \mathrm{m}^{2}$. When excited from the side by a ultra-violet emitting light emitting diode (LED) and looked at through the treated surface, the parts on which the photonic crystal structure exists appear brighter, hence illustrating a more efficient light extraction (Fig. 7). More work is underway to quantify this improvement and to compare the angular distributions of the extracted photons with and without the photonic crystal.

\section{DISCUSSION}

The first generation of commercial TOF PET scanners has achieved a timing resolution of 550 to $650 \mathrm{ps}$. Breaking the barrier of $500 \mathrm{ps}$ is mandatory for a direct TOF localization of the organ under examination and for improving the rejection of the background generated by other organs.

Practical PET devices require crystals of small cross-section to guarantee a good spatial resolution and sufficient length to convert the $511 \mathrm{KeV}$ gamma rays with a high enough efficiency. The large resulting aspect ratio has a strong influence on the light output as well as on the spread in propagation time of the 
emitted photons. Both factors affect the timing resolution of the crystal.

The photo statistics defines the timing fluctuations of the photo electrons as a function of their rank in the detection process. It results from a combination of the light output (light yield corrected by light transport losses), rise time and decay time. Our study shows that a crystal/photo detector system producing 2200 pe per $511 \mathrm{KeV}$ gamma ray (mean value measured on 1000 pixels of LYSO $2 \times 2 \times 20 \mathrm{~mm}^{3}$ produced for the ClearPEM-Sonic project and measured with a Photonis 2020Q photomultiplier [5]), with $40 \mathrm{~ns}$ decay time and assuming no rise time, would require lowering the detection threshold to 6 pe to achieve a timing resolution of 100 ps limited by photo statistics only. If the light output were to reach 4000 pe or the decay time to be reduced to $20 \mathrm{~ns}$ the detection threshold could be raised to 10 pe.

But an important limitation comes from the rise time, which results itself from the light production mechanism in the scintillator (intrinsic rise time) and the spread in the transit time of the photons from the emission point to the photo detector through different propagation modes. The consequence is a reduced rate of the detected photons in the initial phase of the light pulse, which has the same effect on the timing resolution as a reduced light output. Table I shows that for a crystal having the same light output and decay time as the LYSO crystals mentioned above a 200 ps rise time would imply lowering the detection threshold to one photo electron only to achieve 100 ps timing resolution. Any longer rise time would make this objective impossible, and would require either a higher light output or a shorter decay time. Unfortunately very few reliable data exist yet on the rise time of scintillators. Ongoing measurements should allow us to contribute to a better understanding of the rise time in a large number of known scintillators.

However in properly selected materials a small number of Cerenkov photons are produced by the recoil electrons of a 511 $\mathrm{KeV}$ gamma conversion. The present study shows that these photons can indeed be detected and produce a much narrower distribution than scintillation photons. In a preliminary coincidence measurement we have obtained a coincidence timing resolution of about 400 ps FWHM between 2 LuAG:Ce crystals $2 \times 2 \times 8 \mathrm{~mm}^{3}$, and of $250 \mathrm{ps}$ FWHM, when lowering the detection threshold, between 2 un-doped LuAG crystals of the same dimensions. More work is in progress and will be published soon to measure the detection efficiency of these Cerenkov photons and to understand in more details the potential improvement in the timing resolution as a function of the crystal scintillation properties (light output, rise and decay time).

Finally the use of photonic crystals should enhance the light extraction efficiency by a factor between $50 \%$ and $100 \%$ depending on the scintillator.

\section{CONCLUSION}

Ultimate timing resolution requires a low detection threshold, fast light emission mechanisms and a good control on the light transport and extraction. It is shown that by making use of the Cerenkov emission induced by the recoil electrons from the conversion of $511 \mathrm{keV} \gamma$-rays, and of photonic crystals to extract photons from the crystal with high efficiency one can significantly improve the timing resolution of scintillator-based detectors.

\section{ACKNOWLEDGMENT}

The authors would like to thank Xavier Letartre and Christian Seassal from the Nanotechnology Institute in Lyon for their help and support in preparing the photonic crystal samples.

\section{REFERENCES}

[1] W. W. Moses and S. E. Derenzo, "Prospects for time-of-flight PET using LSO scintillator," IEEE Trans. Nucl. Sci., vol. 46, pp. 474-478, 1999.

[2] K. Parodi, K. F. Pönisch, and W. Enghardt, "Experimental study on the feasibility of in-beam PET for accurate monitoring of proton therapy," IEEE Trans. Nucl. Sci., vol. 52, pp. 778-786, 2005.

[3] B. Henderson and G. F. Imbush, Optical Spectroscopy of Inorganic Solids. London, U.K.: Oxford Science/Clarendon, 2006, p. 160.

[4] F. Powolny, "Characterization of Time Resolved Photo Detector Systems for PET" Ph.D. dissertation, Université de Neuchâtel, Neuchâtel, Switzerland [Online]. Available: http://doc.rero.ch/record/ $12683 ? \ln =$ de

[5] ClearPEM-Sonic [Online]. Available: http://www.cern.ch/cerimed/, a CERIMED pilot project.

[6] Y. Shao, "A new timing model for calculating the intrinsic timing resolution of a scintillator detector," Phys. Med. Biol., vol. 52, pp. 1103-1117, 2007.

[7] P. Dorenbos, "Fundamental limitations and challenging opportunities in the quest for better scintillators," presented at the SCINT2009 Conf. Scintillators and Their Applications, Jeju Island, Korea, Jun. 2009.

[8] W. Lehmann, "Edge emission of n-type conducting $\mathrm{ZnO}$ and CdS," Solid State Electron., vol. 9, pp. 1107-1110, 1966.

[9] S. Derenzo, M. Weber, and M. Klintenberg, "Temperature dependence of the fast, near-band-edge scintillation from $\mathrm{CuI}, \mathrm{HgI} 2, \mathrm{PbI} 2, \mathrm{ZnO}: \mathrm{Ga}$ and CdS:In," Nucl. Instrum. Methods Phys. Res. A, vol. A486, pp. 214-219, 2002.

[10] S. Seifert, R. Vinke, H. T. van Dam, H. Löhner, P. Dendooven, F. J. Beekman, and D. R. Schaart, "Ultra precise timing with SiPM-based TOF PET scintillation detectors," in Proc. Nuclear Science Symp. Conf. Rec., Orlando, Fl, 2009, (J01-4).

[11] M. Conti, L. Eriksson, H. Rothfuss, and C. L. Melcher, "Comparison of fast scintillators with TOF PET potential," IEEE Trans. Nucl. Sci., vol. 56, no. 3, pp. 926-933, Jun. 2009.

[12] M. Kronberger, E. Auffray, and P. Lecoq, "Probing the concepts of photonic crystals on scintillating materials," IEEE Trans. Nucl. Sci., vol. 55, no. 3, pp. 1102-1106, Jun. 2008.

[13] M. Kronberger, E. Auffray, and P. Lecoq, "Improving light extraction from heavy inorganic scintillators by photonic crystals," in Proc. IEEE Nuclear Science Symp. Conf. Rec., Dresden, Germany, 2008, pp. 3914-3919. 